\title{
Spectrum of Lesions on Upper Gastrointestinal Endoscopy and Its Correlation with Histopathological Evaluation
}

\author{
Preeti Rajendra Sahu ${ }^{1}$, Kishor Madhukar Hiwale², Sunita Jayant Vagha ${ }^{3}$, Samarth Shukla ${ }^{4}$ \\ ${ }^{1}$ Department of Pathology, Jawaharlal Nehru Medical College, Acharya Vinoba Bhave Rural Hospital, \\ Sawangi (Meghe), Wardha, Maharashtra, India. ${ }^{2}$ Department of Pathology, Jawaharlal Nehru Medical \\ College, Acharya Vinoba Bhave Rural Hospital, Sawangi (Meghe), Wardha, Maharashtra, India. ${ }^{3}$ Department \\ of Pathology, Jawaharlal Nehru Medical College, Acharya Vinoba Bhave Rural Hospital, Sawangi (Meghe), \\ Wardha, Maharashtra, India. ${ }^{4}$ Department of Pathology, Jawaharlal Nehru Medical College, Acharya Vinoba \\ Bhave Rural Hospital, Sawangi (Meghe), Wardha, Maharashtra, India.
}

\section{ABSTRACT}

\section{BACKGROUND}

Upper gastrointestinal (GI) disorders are highly prevalent in the general population and cause significant morbidity. The disease burden leads to impairment in quality of life and considerable healthcare costs. An upper GI endoscopy or EGD (oesophagogastroduodenoscopy) is a procedure to diagnose and treat problems in your upper GI (gastrointestinal) tract. Upper GI endoscopy is often used to diagnose and identify disorders such as GERD (Gastro-Oesophageal Reflux Disease), Barrett's oesophagus, eosinophilic oesophagitis, infectious oesophagitis, oesophageal carcinoma, peptic ulcer diseases, gastric carcinoma, celiac disease or inflammatory bowel diseases, and sometimes as a treatment modality in cases of foreign body obstruction, for performing laser therapy, for percutaneous gastrostomy tube, to control variceal bleeding and so on. GI symptomatology tends to have a varied range and clinical presentations are usually overlapping with no clear-cut specificity of any symptom with any of the upper GI disorders. The workup in determining the aetiology of upper GI complaints includes laboratory investigations and various imaging modalities. An endoscope can be used to take tissue samples (biopsies). Thus, it remains an important tool in the diagnosis. Correlation of Upper GI Endoscopic findings along with histopathological diagnosis is very effective and necessary to reach a definitive diagnosis. In this review, we describe the available evidence in the literature, including the more recent published guidelines, on when and from where the endoscopic biopsies should be done in upper GI, focusing on the precise diagnosis of the most common gastrointestinal diseases. Therefore, this study was planned to study the spectrum of upper gastrointestinal tract diseases by correlating endoscopic and histopathological findings.

\section{KEY WORDS}

Upper GI Endoscopy, Oesophageal Biopsy, Gastric Biopsy, Duodenal Biopsy
Corresponding Author: Dr. Preeti Rajendra Sahu, Department of Pathology, Jawaharlal Nehru Medical College, Acharya Vinoba Bhave Rural Hospital, Sawangi, Meghe, Wardha, Maharashtra, India.

E-mail: preetisahu9811@gmail.com

DOI: $10.14260 /$ jemds/2020/498

How to Cite This Article:

Sahu PR, Hiwale KM, Vagha SJ, et al. Spectrum of lesions on upper gastrointestinal endoscopy and its correlation with histopathological evaluation. J. Evolution Med. Dent. Sci. 2020;9(32):2301-2306, DOI: $10.14260 /$ jemds/2020/498

Submission 22-04-2020,

Peer Review 29-06-2020,

Acceptance 07-07-2020,

Published 10-08-2020.

Copyright (C) 2020 JEMDS. This is an open access article distributed under Creative Commons Attribution License [Attribution 4.0 International (CC BY 4.0)] 


\section{BACKGROUND}

Gastrointestinal (GI) diseases present with symptoms of abdominal pain, heartburn, diarrhoea, nausea, vomiting, flatulence, difficulty in swallowing, dysphagia, bloated abdomen, significant weight loss, fullness after having very little meal, and melena. Taking into account of just the upper GI bleed incidence, it ranges from 50 to $150 / 100,000$ population annually, and time trend analyses suggest that aged people constitute an increasing proportion of those presenting with acute upper GI bleed. ${ }^{1}$

An upper GI endoscopy or oesophagogastroduodenoscopy/ EGD aids in diagnosing and treating disorders of upper GIT. Endoscopy gives a visual look of GI mucosa and allows tissue sampling, for further assessment by pathologist. Abnormal endoscopic appearance indicates a disease, where biopsy will confirm. ${ }^{2}$ Histopathological examination (HPE) is the best confirmatory tool to confirm and find the diagnosis. ${ }^{3}$

Various lesions affecting THE GIT are classified organ wise i.e. oesophageal, gastric and duodenal lesions. Clinical history remains central in evaluating oesophageal symptoms. Chief oesophageal symptoms are pyrosis, reflux, chest pain, dysphagia and odynophagia. Heartburn/pyrosis, is most frequent intermittent oesophageal symptom, presenting as an uneasiness/ burning sensation in retrosternum radiating toward neck. It occurs mostly after eating/while lying recumbent. 4

\section{Eosinophilic Oesophagitis}

Eosinophilic oesophagitis (EoE) is disease of chronic inflammation, caused due to lodgement of food particles and allergic syndromes. It occurs predominantly in males. Characteristic endoscopic findings seen are rings, linear furrows, and white plaques. Endoscopy finding varies depending on which stage the disease is in. Features suggesting mucosal inflammation are frequently seen in young patients, whereas narrowing or strictures are seen more in elderly. It is advisable to take 2-4 biopsies of both proximal and distal oesophagus whenever the patient is suspected of eosinophilic oesophagitis. ${ }^{5}$ The key findings is $\geq 15$ eosinophils/HPF i.e high power field within the squamous epithelium forming small aggregates known as eosinophilic microabscesses. ${ }^{6}$ It is associated with eosinophil degranulation and basal cell hyperplasia. Submucosal fibrosis present in chronic cases. Diagnosis of EoE is based on allergic history, oesophageal $\mathrm{pH}$ probes (nonacidic) and the gold standard for diagnosis is HPE after endoscopic biopsy.

\section{Infectious Oesophagitis}

Increased usage of immuno-suppressants having transplant and chronic inflammatory diseases, chemotherapy patients; infections are caused commonly by cytomegalovirus (CMV), human herpesvirus (HSV) and candida. ${ }^{5}$

\section{Candida}

Candida species colonises approximately $20 \%$ in healthy humans. Immunocompromised hosts like in AIDS, cancer patients or those who are in steroid therapy are at risk. The most common causative organism is C. albicans. Patient complains of odynophagia/dysphagia. Diagnosis is achieved by endoscopy, culturing the brushings from mucosa and HPE of mucosa of oesophagus. Approximately $2 / 3^{\text {rd }}$ have signs of oral thrush. At present the most sensitive and specific test to diagnose is oesophagogastroduodenoscopy with brushings or biopsy. On endoscopy patchy, whitish plaques covering a friable, erythematous mucosa. In severe infections, ulceration can be seen. ${ }^{7}$ In suspected cases, multiple biopsies should be taken from the involved area. For biopsy to confirm candida, yeasts and pseudohyphae invading mucosa are must.

\section{Herpes Simplex Virus}

HSV-1 usually affects seen in immunocompromised conditions such as organ or bone marrow transplantation. Endoscopically, punched out appearance is seen with well circumscribed ulcers having raised margins. Cytopathic effects caused by virus are mostly seen at the edges or margin of ulcer, thus it is recommended to perform biopsy from these areas. ${ }^{7}$ HSV infects mainly the squamous epithelial cells. Herpes simplex virus show the presence of Cowdry Type- A inclusion bodies. On H\&E, they appear as droplet-like masses of acidophilic substance surrounded by clear halos within nuclei, with margination of chromatin on the nuclear membrane. ${ }^{8}$ PCR (Polymerase Chain Reaction) or culture also helps in reaching the diagnosis. ${ }^{5}$

\section{Cytomegalovirus}

It is most common cause of viral oesophagitis in advancing AIDS patient. It infects predominantly columnar cells, and is seen in immunocompromised states. Endoscopically, the characteristic finding in oesophagus is extensive ulcer formation. It can be either single or multiple ulcers. Majority of these ulcers are seen in distal oesophagus. ${ }^{7}$ It is advisable to take multiple biopsies from ulcerative base. On histopathology the most characteristic feature cytoplasmic which is basophilic and eosinophilic intranuclear inclusion bodies, giving as owl's eye appearance. In immunocompromised hosts, atypical inclusions have been reported more often than typical Cowdry inclusions. ${ }^{9}$ Minimum of 3 biopsy samples shows sensitivity of at least $80 \%$. In finding out CMV, PCR has a greater sensitivity as compared with histopathological findings ${ }^{5}$

\section{Gastroesophageal Reflux Disease (GERD)}

Recent studies showed prevalence of GERD in India between $8 \%$ to $24 \%$, compared with the Western world. ${ }^{10}$ The most common complication of GERD is oesophagitis. ${ }^{5}$ Heart burn, chest pain and odynophagia are common symptoms. Early endoscopy at initial stage of presentation is recommended whenever alarming symptoms of complicated disease are felt or patients who at risk for Barrett's oesophagus. Two grading systems used for severity of erosive reflux oesophagitis is Savary-Miller endoscopic classification and Los-Angeles grading. The Savary-Miller classification is commonly done but it has variable utilisation along with interpretation. MUSE classification (stands for Metaplasia, Ulceration, Stricturing, and Erosions) provides clear definitions of the relevant endoscopic features, and it is based on a standardized report 
form, which allows the endoscopist to make a clear record of oesophagitis severity. Histomorphological finding shows $\geq 15$ eosinophils per HPF, distribution of eosinophil is concentrated distally, whereas scattered single eosinophils is most common pattern of eosinophilia. The most worrisome histopathological complication of GERD is the Barrett's oesophagus, especially when associated with oesophageal adenocarcinoma (20 times more risk). ${ }^{6}$

\section{Barrett's Oesophagus}

Barrett's Oesophagus (BO) is an example of metaplasia where stratified squamous epithelium gets replaced by columnar epithelium predisposing it for development of carcinoma. It is visualised via endoscope and then confirmed by histopathological examinations. Endoscopically, the characteristic appearance is of salmon pink coloured oesophageal mucosa extending down joining the mucosa of stomach. American Gastroenterological Association (AGA) suggested endoscopy should be done to screen for Barrett's oesophagus in cases having various risk factors associated with oesophageal adenocarcinoma "age $\geq 50$ years, male gender, white race, chronic GERD, hiatal hernia, elevated BMI and elevated intra-abdominal distribution of body fat. ${ }^{7}$ Prague classification should be used when describing the degree of Barrett's oesophagus, taking proximal gastric folds as a reference. Seattle protocol have higher rate of success in diagnosing endoscopically, especially in detecting dysplastic changes.

It recommends performing biopsy in 4 quadrants at each 2 $\mathrm{cm}$. the protocol for follow-up is as follows

- $\quad$ BO without dysplasia it is advisable to take biopsies of 4 quadrants at each $2 \mathrm{~cm}$ every $3---5$ years

- $\quad$ BO with low grade dysplasia it is recommend taking biopsies of 4 quadrants at each $1-2 \mathrm{~cm}$ every 6---12 months.

- $\quad$ BO with high-grade dysplasia it is recommended to take biopsies of 4 quadrants each $1 \mathrm{~cm}$ every 3 monthly, in the absence of treatment for its eradication.

Short segments $(<3 \mathrm{~cm})$ without intestinal metaplasia should not be followed after a second endoscopy confirming the absence of metaplasia. ${ }^{11}$ In India, prevalence is 2.4 $23.6 \% .^{12}$

\section{Carcinoma Oesophagus}

It is $8^{\text {th }}$ ranked cancer worldwide, with an estimation of approximately 450,000 new cases in annually and $6^{\text {th }}$ ranked cause of mortality from cancer with an estimated 400,000 deaths. ${ }^{13}$ The overall five-year survival is $15-20 \%{ }^{14}$ Nearly $80 \%$ are malignant and $>90 \%$ of these are squamous cell carcinomas (SCCs) or adenocarcinomas. ${ }^{15}$

Globally, $80 \%$ of oesophageal SCC (ESCC) occurs in developing countries making it most predominant histopathological subtype. The global incidence of squamous cell cancer represents $87 \%$ of all cases of oesophageal cancer in $2012 .{ }^{14}$ Less than $1 \%$ to $2 \%$ of all oesophageal cancers are sarcomas or small cell carcinomas. ${ }^{16}$ Male predominance is noted as compared to female in the oesophageal squamous cell carcinoma. ${ }^{13} \mathrm{An}$ overall global assessment indicated a male tofemale ratio of 4.4. Squamous dysplasia is precursor lesion for development of ESCC. ${ }^{17}$ Patients usually comes with difficulty to swallow solid which increases to liquids as well. Two major types of oesophageal carcinoma: squamous cell carcinoma and adenocarcinoma.

Mostly SCCs affects the middle third of oesophagus, followed by upper and lower third. Pathologically, it presents as polypoid mass, flat or ulcerated lesions. About $65 \%$ are men of peak age ranging from 60-74 years. ${ }^{15}$ Endoscopically ESCC seen in three classical forms- most common is polypoidal mass, followed by mass having depressed ulceration in centre and lastly diffusely infiltrating one. ${ }^{7}$ Adenocarcinomas are $2^{\text {nd }}$ common malignant tumour of oesophagus. About $85 \%$ of oesophageal adenocarcinoma patients are men.15 Endoscopically adenocarcinoma presents as an infiltration having narrowed lumen with or without associated mass. Also shows nodularity with friable and eroded mucosa. Endoscopy with multiple biopsies increases diagnostic yield.

\section{Peptic Ulcer Disease}

Within the upper GI, acute bleeding is the commonest causes with which patients present to casualty worldwide.18 GI bleeding reports mortality of $5-15 \% .^{19}$ Leading reason for acute upper GI Bleed is peptic ulcer disease. In a recent study, it was shown that gastroduodenal ulcers were responsible for upper gastrointestinal bleeding in $50 \%$ of cases. ${ }^{20}$ Upper GI Bleeding can occur from multiple different sites in the GIT. On the basis of endoscopic morphology ulcers are divided into benign ulcers and malignant ulcers. Benign ulcers have a smooth, regular, rounded edges, with a flat base having exudate. The white ulcerative base is filled with fibrinous granulation tissue.

\section{Malignant Ulcer}

Have an ulcerative growth with nodular appearing folds and asymmetrical overhanging, nodular margin suggesting a malignant ulcer. The chances of malignancy increase with large gastric ulcers. ${ }^{7}$ Duodenal ulcer uncommonly have malignant features while gastric ulcers frequently have malignant ulcerated neoplasms. ${ }^{5}$ In approximately $20 \%$ of patients, endoscopic findings cannot differentiate between benign and malignant ulcer. For diagnosing majority of carcinomas minimum of four to six biopsies from ulcerative margin have given a good result. Even if the ulcer looks benign, then also multiple endoscopic biopsies should be done as it can be malignant. 7 Helicobacter pylori ( $\mathrm{H}$. pylori) is a microaerophilic Gram -ve bacterium. H. pylori causes active chronic gastritis, peptic ulcer, dyspepsia, cancer and MALT lymphoma. ${ }^{21}$ The risk of peptic ulcer is nearly 10 fold higher in patients with non-atrophic Helicobacter pylori positive gastritis than in those with a normal stomach, and the risk is increased further (2-3 fold) when there is antral atrophy. It is the major cause of chronic gastritis. ${ }^{17}$

For histopathological diagnosis protocol of Sidney is followed for taking endoscopic biopsy

- From both lesser and greater curvature antrum and body should be sampled, biopsy from antrum should be not beyond 2---3 $\mathrm{cm}$ of pylorus and for taking from body of stomach it should be $8 \mathrm{~cm}$ from cardia. 
- From incisura angularis: first from incisura angularis, then from greater curvature of body and antrum. ${ }^{5}$

- $\quad$ Routine H\&E stain, silver methamine and rapid urease breath test while performing endoscopy can detect $\mathrm{H}$. pylori.

\section{Chronic Gastritis Type B}

It is commonly encountered type of gastritis where $\mathrm{H}$. pylori pathogen plays a major role in its genesis. Historically it was denoted as predominantly antral, approximately transformation into pangastritis depends on time, which occurs at 15--20 years. To assess the presence and degree of dysplasia or neoplastic changes biopsies are done. The two optional approaches generally used for suspected case of type B chronic gastritis are:

- 7 biopsies randomly taken from fundus, body and antrum of stomach.

- 12 biopsies protocol: 4 biopsies from each quadrant of antrum, 2 biopsies from incisura angularis, 4 biopsies from body and 2 biopsies from cardia. The protocol for environmental metaplastic atrophic gastritis should be sufficient for the histological diagnosis of $\mathrm{H}$. pylori infection. ${ }^{5}$

\section{Autoimmune Atrophic Metaplastic Gastritis or Chronic Gastritis Type A}

Autoimmune atrophic metaplastic gastritis involves mainly fundus and body of stomach, excluding antrum. Conventionally, it is linked with pernicious anaemia due to circulating anti-parietal cells and/or anti-intrinsic factor antibodies which are present. It has an increased risk of adenocarcinoma and carcinoid tumours. The biopsies should be performed at ulcers, nodules, polyps or growth in order to exclude malignancy.

\section{Gastric Carcinoma}

Is $3^{\text {rd }}$ leading cause of cancer-causing mortality worldwide (723,000 deaths, $8.8 \%$ of total) according to GLOBOCAN project results in 2012, published by the International Agency for Research Cancer. In India, the occurrence of gastric carcinoma (GC) is low. As compared to global age-adjusted rate (AAR 4.1-95.5), in India AAR for carcinoma stomach is 3.0-13.20.22 Over $90 \%$ of stomach cancers are adenocarcinoma. Endoscopic surveillance is must once or twice/ year for high risk patients of gastric carcinoma (history of gastric carcinoma in family, FAP, Li-Fraumeni syndrome, BRCA2 mutations, HNPCC syndrome, Peutz-Jeghers syndrome). ${ }^{23}$

Lauren classification divides into 2 important types, intestinal and diffuse type. Intestinal type occurs often in distal stomach, preceded by a long-standing precancerous lesion. Diffuse type occurs young patients. For diagnosis of gastric carcinoma oesophagogastroduodenoscopy has $95 \%$ diagnostic accuracy. Initially gastric carcinomas may look like slight polypoid protrusion, superficial plaque, discoloration of mucosa or ulcer. The primary method to obtain tissue from a suspected lesion is via endoscopy. For taking biopsy of an ulcerative lesion a minimum of 6 specimens must be taken surrounding the lesion due to malignant transformations which can occur. Gastric cancers can be diagnosed at an earlier stage if the upper endoscopy is performed initially, when patients have GI complains. Diagnosing linitis plastic based just on endoscopy is not easy. As tumours infiltrate submucosa and muscularis propria, there are chances of false negative, if biopsy is performed superficially. ${ }^{7}$ Lymphoma characterizes $5 \%$ of all gastric malignancies are which are primary, among them extra-nodal marginal zone B- cell lymphomas are very frequent. In gut they are often referred to lymphomas of mucosa- associated lymphoid tumours, MALTomas. ${ }^{24}$

\section{Duodenum}

The etiological factors for malabsorption syndrome differ according to age of patients and their location. Celiac sprue and inflammatory bowel disease like Crohn's disease are frequent causes of malabsorption in developing nations, like India. ${ }^{25}$ Histopathological examination of biopsy is one of the major steps in assessing patients which are suffering from malabsorption. Duodenum is most accessible site thus it is commonly used for biopsy. Performing multiple biopsy from $2^{\text {nd }}$ part of duodenum increases the accuracy of diagnosing a disease.

\section{Celiac Disease}

Celiac disease (CD) also known as gluten-sensitive enteropathy, celiac sprue, or non-tropical sprue. Primarily associated with the HLA-DQ2 allele. It occurs in both children and adults. Around $50 \%$ of cases suffering with celiac sprue show typical clinical features, such as Vitamin B12 Deficiency, weight loss, diarrhoea, abdominal pain. Duodenal biopsy is done with a positive IgA endomysial or transglutaminase antibody; or when clinical symptoms are highly suggestive even in the absence of negative antibody.

Modified Marsh-Oberhuber classification is currently used is of 4 types: -

1. Pre-infiltrative, normal mucosa with $<30$ IELs (intra epithelial lymphocytes) per 100 enterocytes.

2. Infiltrating type, normal villous-crypt architecture (normally $>3: 1)$ and high IELs $(\geq 30$ IELs / 100 enterocytes).

3. Infiltrative-hyperplastic type, normal villous architecture and crypt hyperplasia with high IELs $(\geq 30$ IELs).

4. Flat mucosal type of celiac disease- Further divided into 3 groups based on atrophy of the villous.

3a- Mild atrophy with villous to crypt ratio of $<3: 1$ or 2:1 and high IELs ( $\geq 30$ IELs).

3b: Marked atrophy with villous to crypt ratio of $<1: 1$ and high IELs ( $\geq 30$ IELs).

3c- Total atrophy with completely flat mucosa and high IELs.

5. Atrophic type, having flat mucosa with just few crypts seen. ${ }^{26}$

Biopsies should be done whenever there is positive anticeliac antibodies. ${ }^{27}$ From both duodenal bulb and distal duodenum nearly 4- 6 biopsy samples should be taken. On endoscopy the duodenal mucosa appears atrophic with loss of folds, fissures and nodularity are seen. Multiple biopsies should be performed in $2^{\text {nd }}$ and $3^{\text {rd }}$ part of the duodenum. 
Video capsule endoscopy shows good sensitivity and excellent specificity for the detection of villous atrophy in patients with suspected celiac disease. ${ }^{7}$

\section{Crohn's Disease}

Crohn's disease is idiopathic, chronic regional transmural inflammation of the GIT. Peak age prevalence is 30-39 years. Most common symptom is chronic diarrhoea. Primary peripheral arthritis (33\%) is most frequent extraintestinal manifestation. ${ }^{27}$ It involves distal small intestine and right side of colon and in 20-30\% cases only small bowel is involved.

The characteristic histopathological findings are

- Lamina propria with increased plasma cells and lymphocytes, architectural destruction with patchy, neutrophilic inflammation comprising neutrophilic cryptitis or crypt abscesses.

- Skip lesions consisting of focal, patchy erosions or ulcers, vertical fissures and fistulas.

- $\quad$ "Skip lesions" - Mucosal fragments from the same level of the colon have different HPE findings.

- Granulomas with multiple lymphoid aggregates.

- Submucosal fibrosis and neuromuscular hyperplasia of submucosa.

The fissures are often knife-like covered by epithelioid histiocytes, and giant cells. ${ }^{28}$ On endoscopy, linear ulcers, aphthous ulcers, round or irregular ulcers, pseudopolyps, cobble stoning, stricture or stenosis are seen. ${ }^{7}$

\section{CONCLUSIONS}

Upper GI endoscopy is an important diagnostic modality as well as a tool in the management of GI diseases in recent years. Besides conventional investigations like barium swallow, meal or follow through, advances in imaging technology (ultrasound, capsule endoscopy, etc.) have helped GI specialists in detecting and managing disorders early in the course throughout the GI tract. Promoting use of endoscopy as a screening test in the above-mentioned conditions will be a major step towards reducing GI morbidity and mortality. To realize the real potential of the correlation between endoscopy and histopathological findings, this review article reviews the endoscopic indications along with their findings on endoscopy and on histopathology.

Financial or Other Competing Interests: None.

\section{REFERENCES}

[1] Thomopoulos KC, Vagenas KA, Vagianos CE, et al. Changes in aetiology and clinical outcome of acute upper gastrointestinal bleeding during the last 15 years. Eur J Gastroenterol Hepatol 2004;16(2):177-82.

[2] Teriaky A, AlNasser A, McLean C, et al. The utility of endoscopic biopsies in patients with normal upper endoscopy. Can J Gastroenterol Hepatol 2016;2016:1-7.
[3] Swarnkar M, Jain SC. Heterotopic subserosal pancreatic tissue in jejunum-an incidental rare finding. JKIMSU 2017;6(4):105-8.

[4] Kahrilas PJ, Hirano I. Diseases of the oesophagus. In: Kasper D, Fauci A, Hauser S, et al, eds. Harrison's principles of internal medicine. 19th edn. McGraw-Hill Medical 2015.

[5] Peixoto A, Silva M, Pereira $P$, et al. Biopsies in gastrointestinal endoscopy: when and how. GE Port J Gastroenterol 2015;23(1):19-27.

[6] Conner JR, Kirsch R. The pathology and causes of tissue eosinophilia in the gastrointestinal tract. Histopathology 2017;71(2):177-99.

[7] Amornyotin S. Endoscopy of GI tract. Intech Open 2013: p. 362.

[8] Kulkarni M, Agrawal T, Dhas V. Histopathologic bodies: an insight. J Int Clin Dent Res Organ 2011;3(1):43-7.

[9] Reddy N, Wilcox CM. Diagnosis and management of cytomegalovirus infections in the GI tract. Expert Rev Gastroenterol Hepatol 2007;1(2):287-94.

[10] Bhatia SJ, Reddy DN, Ghoshal UC, et al. Epidemiology and symptom profile of gastroesophageal reflux in the Indian population: report of the Indian Society of Gastroenterology Task Force. Indian J Gastroenterol 2011;30(3):118-27.

[11] Loughrey MB, Johnston BT. Guidance on the effective use of upper gastrointestinal histopathology. Frontline Gastroenterol 2014;5(2):88-95.

[12] Wani IR, Showkat HI, Bhargav DK, et al. Prevalence and risk factors for barrett's oesophagus in patients with GERD in Northern India; do methylene blue directed biopsies improve detection of Barrett's oesophagus. Compared the conventional method? Middle East J Dig Dis 2014;6(4):228-36.

[13] Arnold M, Soerjomataram I, Ferlay J, et al. Global incidence of oesophageal cancer by histological subtype in 2012. Gut 2015;64(3):381-7.

[14] Abbas G, Krasna M. Overview of oesophageal cancer. Ann Cardiothorac Surg 2017;6(2):131-6.

[15] Li YZ, Wu PH. Conventional radiological strategy of common gastrointestinal neoplasms. World J Radiol 2015;7(1):7-16.

[16] Napier KJ, Scheerer M, Misra S. oesophageal cancer: a review of epidemiology, pathogenesis, staging workup and treatment modalities. World J Gastrointest Oncol 2014;6(5):112-20.

[17] Rosai J. Rosai and Ackerman's surgical pathology. $9^{\text {th }}$ edn. Edinburgh: Mosby 2004.

[18] Kankaria AG, Fleischer DE. The critical care management of nonvariceal upper gastrointestinal bleeding. Crit Care Clin 1995;11(2):347-68.

[19] Terdiman JP. Update on upper gastrointestinal bleeding. Basing treatment decisions on patients' risk level. Postgrad Med 1998;103(6):43-64.

[20] van Leerdam ME, Vreeburg EM, Rauws EAJ, et al. Acute upper GI bleeding: did anything change? Time trend analysis of incidence and outcome of acute upper GI bleeding between 1993/1994 and 2000. Am J Gastroenterol 2003;98(7):1494-9.

[21] Wroblewski LE, Peek RM, Wilson KT. Helicobacter pylori and gastric cancer: factors that modulate disease risk. Clin Microbiol Rev 2010;23(4):713-39. 
[22] Rastogi T, Devesa S, Mangtani P, et al. Cancer incidence rates among South Asians in four geographic regions: India, Singapore, UK and US. Int J Epidemiol 2008;37(1):147-60.

[23] Sitarz R, Skierucha M, Mielko J, et al. Gastric cancer: epidemiology, prevention, classification, and treatment. Cancer Manag Res 2018;10:239-48.

[24] Sagaert X, De Wolf-Peeters C, Noels H, et al. The pathogenesis of MALT lymphomas: where do we stand? Leukemia 2007;21(3):389-96.

[25] Ranjan P, Ghoshal UC, Aggarwal R, et al. Etiological spectrum of sporadic malabsorption syndrome in northern Indian adults at a tertiary hospital. Indian J Gastroenterol 2004;23(3):94-8.
[26] Bao F, Green PHR, Bhagat G, et al. An update on celiac disease histopathology and the road ahead. Arch Pathol Lab Med 2012;136(7):735-45.

[27] Ha F, Khalil H. Crohn's disease: a clinical update. Ther Adv Gastroenterol 2015;8(6):352-9.

[28] Rendi M, Younes M. Crohn disease pathology. https://emedicine.medscape.com/article/1986158overview\#a7 